\title{
Fantasien der Auslöschung: Christian Krachts Romane Faserland und 1979
}

Christine Lehmann*

\begin{abstract}
At the end of the nineties a new stream of "pop-literature" was discussed by German philology. The author Christian Kracht is often mentioned as one of the founders of this genre. In this article two novels of Christian Kracht will be discussed: Faserland and 1979. While many critics consider Faserland as a prototype of "pop-literature", 1979 already seems to announce the end of this period. Here, the two novels are interpreted and analyzed with respect to their typical elements of "pop-literature".
\end{abstract}

Keywords: Pop-literature; Kracht

Zusammenfassung: Ende der 90er Jahre diskutiert die germanistische Literaturwissenschaft über eine „,neue deutsche Popliteratur“. Der Autor Christian Kracht wird als ein Begründer dieser literarischen Strömung gesehen. Der vorliegende Beitrag beschäftigt sich mit Krachts Romanen Faserland (1995) und 1979 (2001). Während Faserland oft als Prototyp der Popliteratur angesehen wird, scheint 1979 eher einen Ausstieg aus diesem noch schwer zu definierenden Genre anzudeuten. Beide Romane werden in diesem Beitrag interpretiert und hinsichtlich ihrer popliterarischen Elemente diskutiert.

Stichwörter: Popliteratur; Kracht

Die Autorin ist zur Zeit Sprachassistentin des DAAD an der Universidade Federal do Ceará in Fortaleza; sie hat ihren Magisterabschluss in deutscher Literatur an der Universität Leipzig gemacht. lehmann.z@web.de 
Resumo: No final dos anos 90, a ciência da literatura germanística discute sobre uma "nova literatura alemã pop". O autor Christian Kracht é considerado o fundador dessa corrente literária. A presente contribuição ocupa-se com os romances de Kracht: Faserland e 1979. Enquanto Faserland é visto freqüentemente como protótipo da literatura pop, 1979 parece sugerir antes uma saída desse gênero ainda defícil de definir. No presente trabalho os dois romances são interpretados e discutidos no que se refere aos seus elementos popliterários.

Palavras-chave: Literatura Pop; Kracht

\section{Einleitung}

Junger Schnösel Mitte Zwanzig, dandyhaft in Kleidung und Gebaren, lässt sich vor Langeweile einmal von Norden nach Süden durch Deutschland bis in die Schweiz treiben, ergeht sich vorwiegend darin, den Vorzug von grünen gegenüber blauen Barbourjacken oder Brooks Brothers- gegenüber Ralph Lauren-Hemden zu verkünden und tut überdies seinen Weltekel kund. Diesen Eindruck erweckt zunächst der Protagonist beim Lesen des Romans Faserland von Christian Kracht. Beeinflusst durch diverse Meldungen in den Medien über Kracht, auch über seine Selbstinszenierungen zusammen mit anderen so genannten Popliteraten, waren viele Kritiker geneigt, den Autor mit dem Ich-Erzähler gleichzusetzen und die Antipathien gegenüber dem Ich-Erzähler auf den Autor zu projizieren. Ende der 90er-Jahre wird die Diskussion über Pop und Literatur in den Feuilletons lauter und Verlage wie Kiepenheuer \& Witsch beginnen die ,neue deutsche Popliteratur" (vgl. die „alte“ der 70er-Jahre) in großen Auflagenzahlen zu vermarkten. Neu sind nicht die Erzähltechniken der jungen Autoren, sondern dass diese als erfolgreiche Medienschaffende, etwa als Journalisten oder Gagschreiber für Fernsehshows, als DJs oder Ähnliches arbeiten. Von den Kritikern werden sie sogleich der Hochstapelei bezichtigt. Es heißt, die Autoren wollen sich nur ,als Ästheten, Meinungsführer, ja als Popstars profilieren“ (HeIlbrunn 2001: 16). Die Literatur sei für sie ausschließlich eine Karriereoption, die Texte zeigen ,reaktionäres Schnöseltum ohne jeden Biß“ (ZIEGLER 1995). Hinzu kommt der Vorwurf, sie würden dem Thema der deutschen Vergangenheit mit Gleichgültigkeit und Desinteresse begegnen. Vielleicht suchen einige Popliteraten aber auch nach einem 
eigenen Umgang mit der Geschichte ihres Landes. Zu dieser Auseinandersetzung gehört, dass sie gerade die Werte ihrer Eltern in Frage stellen, der so genannten 68er-Generation, die die Schuld „der Deutschen“ in der Hitlerzeit immer wieder thematisierte. Kokett behauptet der Autor Florian IlLIES (2000: 155): „Außerdem sind wir die erste Generation, die wieder über Polenwitze lachen kann, ohne gleich an den Polenfeldzug von 1939 denken zu müssen.“

Mit Erscheinen seines Debütromans Faserland wird Christian Kracht im Feuilleton, wie auch in der beginnenden wissenschaftlichen Auseinandersetzung als Begründer der neuen Popliteratur angesehen. Mittlerweile scheint diese aber wieder aus dem Fokus der öffentlichen Diskussion geraten zu sein: „Seltsam parallel zur New Economy verlief ihre große Zeit etwa von 1995 bis 2000.“ (Freund 2003) Bereits Krachts zweiter Roman 1979, der im Herbst 2001 erscheint, klingt nach Verunsicherung, nach Auslöschung und Untergang. Der Roman, der am Vorabend der islamistischen Revolution 1979 in Teheran sowie an den Schauplätzen Tibet und China spielt, verstörte Leser und Kritiker gleichermaßen, geriet er doch in die Nähe einer diffusen literarischemotionalen Entsprechung zu den omnipräsenten Bildern des 11. September 2001. Es scheint geradezu, als wäre dieser Roman bereits ein Abgesang auf die Popliteratur, angestimmt durch den besten Popliteraten höchst selbst.

Im vorliegenden Beitrag, der aus meiner Magisterarbeit hervorgeht, ist das Kapitel 1 der Interpretation des Romans Faserland gewidmet. Nach einer Inhaltsangabe (1.1) wird das Wesen des Protagonisten beschrieben (1.2), die Ich-Erzählperspektive thematisiert (1.3) und die Verwendung von Markennamen untersucht (1.4). Im Kapitel 2 werden der PopliteraturCharakter von Faserland überprüft und einige Interpretationsansätze kurz diskutiert. In Kapitel 3 wird der Roman 1979 interpretiert. Nach einer Inhaltsangabe (3.1) und der Beschreibung des Protagonisten (3.2) folgen die Analyse der verbliebenen Popelemente (3.3), Hinweise auf intertextuelle Verweise (3.4) und die Beleuchtung des Motivs der Auslöschung (3.5). 


\section{Der Roman Faserland \\ 2.1 Eine Reise durchs "Fa(th)erland"}

Acht Kapitel erzählen von der acht Tage dauernden Reise durch Deutschland in die Schweiz. Das erste Kapitel beginnt unvermittelt:

Also, es fängt damit an, dass ich bei Fisch-Gosch in List auf Sylt stehe und ein Jever aus der Flasche trinke. Fisch-Gosch, das ist eine Fischbude, die deswegen so berühmt ist, weil sie die nördlichste Fischbude Deutschlands ist. [...] Weil es ein bisschen kalt ist und Westwind weht, trage ich eine Barbourjacke mit Innenfutter. (FL: 9).

Der Protagonist findet die Insel ,eigentlich superschön“ (FL: 11) und sagt am Ende des ersten Kapitels doch: „Ich glaube ich werde nicht mehr nach Sylt fahren." (FL: 19) Sollte hier schon feststehen, dass er sich von diesem Land verabschiedet, es nur noch einmal durchquert, um sich seiner Entscheidung zu versichern? Die Menschen, denen er begegnet, scheinen ihm das Dasein so richtig zu verderben. Auch im ICE, mit dem er im zweiten Kapitel nach Hamburg zu seinem Freund Nigel fährt, ist „alles so transparent, ich weiß nicht, ob ich mich da richtig ausdrücke, jedenfalls ist alles aus Glas und aus so durchsichtigem türkisen Plastik, und es ist irgendwie körperlich unerträglich geworden.“ (FL: 20 f.) Die Schauplätze der Handlung verlässt er meist fluchtartig und überlässt dem Zufall, welches das nächste Ziel seiner Reise sein soll. So steigt der Erzähler, nachdem er aus Frankfurt in Richtung Karlsruhe abgereist ist, bereits in Heidelberg aus dem Zug, nur weil Mathias Horx, „so ein Trendforscher“, plötzlich auftaucht. Die besuchten Städte sind austauschbar und werden nur oberflächlich wahrgenommen. Die Eindrücke des Protagonisten wirken wie ,zerfasert“, einmal authentisch die Realität beschreibend, dann wieder wie aus Distanz erzählt und mit Reflexionen über deutsche Geschichte durchsetzt: „Old Heidelberg, Old Heidelberg. Hier steige ich aus. [...] So könnte Deutschland sein, [...] wenn die Juden nicht vergast worden wären. Dann wäre Deutschland so wie das Wort Neckarauen." (FL: 81) Gerade durch die eingeschobenen Reflexionen gleicht die Reise einer Sinnsuche. Das in der Schweiz spielende letzte Kapitel kann als Schlüssel für das Verständnis der Reise gesehen werden. Es scheint, als müsse der Protagonist noch einmal 
zusammenfassen, was über Deutschland erzählt werden muss, über sein Vaterland, von dem er sich abgesetzt hat. Der Erzähler stellt sich vor, was man seinen Kindern weitergeben könnte:

Ich würde ihnen von Deutschland erzählen, von dem großen Land im Norden, [...] von den Menschen würde ich erzählen, [...] die gute Autos fahren müssen und gute Drogen nehmen und guten Alkohol trinken und gute Musik hören müssen, während um sie herum alle dasselbe tun, nur eben ein ganz klein bisschen schlechter. Und das die Auserwählten nur durch den Glauben weiter leben könnten, sie würden es ein bisschen besser tun, ein bisschen härter, ein bisschen stilvoller. (FL: 148)

Wer so über sein Land spricht, kann nur dabei sein, sich zu verabschieden.

\subsection{Der Protagonist - ein einsamer "Held unserer Zeit"}

Vorn hat er „ziemlich lange, hellbraune Haare“ (FL: 13), hinten aber kurz und im Nacken ,sauber ausrasiert“ (FL: 27). Mehr ist nicht zu erfahren: der Held ist äußerlich schemenhaft. Von ihrem inneren Wesen her scheint die Figur von Anfang an vollständig, doch bleibt sie merkwürdig statisch. Als Beschreibungen können kaum die übertreibenden Äußerungen von Varna „ich wäre ein blöder Hippie“ (FL: 58) und von Alexander ,,ich wäre ja ein Nazi und vollkommen unpolitisch“ (FL: 70) gelten. Der Protagonist ist es gerade, der in plötzlichen Ausbrüchen Linke, Ökos und Taxifahrer als „Nazis“"verhöhnt. Gleichzeitig stellt er immer wieder gerne seinen Standesdünkel zur Schau, so wenn er einem Taxifahrer unterstellt, dass „er sauer war, daß wir beide gleich alt sind und ich ein Kiton-Jackett trage und er auf Demos geht" (FL: 26). Der Erzähler ist alles andere als eine positive Identifikationsfigur. Hinter den Wut- und Hasstiraden, dem Gemotze und Geprotze des Erzählers verbirgt sich spürbare Unsicherheit. Hier genießt kein Held seine Jugend und amüsiert sich. Nur oberflächlich betrachtet verhält er sich wie viele Altersgenossen, ist häufig in Kneipen und Bars anzutreffen und geht auf zahlreiche Partys.

Faserlandlässt sich auch als Geschichte von gescheiterten oder scheiternden Freundschaften lesen. Die alten Freunde, die der Held während der Reise 
besucht, enttäuschen ihn oder umgekehrt er sie. So lässt er Rollo im Stich, als dieser am Rande der Geburtstagsparty mit Drogen zugepumpt hilflos am Bodenseeufer steht und weint. Ob er so handelt, weil er selbst schwankt oder schon für sich mit dem Leben abgeschlossen hat? Anzeichen dafür, dass mit ihm ,etwas nicht in Ordnung“ ist, gibt es viele im Text. Das fängt schon bei den unzähligen Malen an, wo es etwa heißt: ,also zünde ich mir eine Zigarette

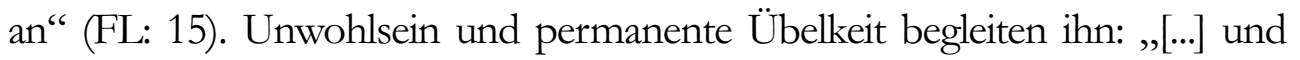
dann muß ich mich übergeben“ (FL: 71). Hinzu kommen angstbesetzte Zukunftsfantasien. Da sind „die großen ungewaschenen Massen aus dem Osten“ (FL: 102). Dennoch ist ihm ein Ost-Mensch in ,,bunten Trainingsanzügen [...] eine Million mal lieber als so ein Understatement-West-Mensch, der irgendwo in einer Einkaufspassage Austern schlürft“ (FL: 102). Von eigenen Zukunftsplänen ist nie die Rede. Lediglich auf eine kindlich zu nennende Art träumt er von einem Leben mit Isabella Rossellini. Diese Träume und Kindheitserinnerungen (,ich merke wie ich fast ein bisschen heulen muss“; FL: 12) stellen einen Kontrast im Erzählfluss dar und spiegeln den tristen gegenwärtigen Gemütszustand des Erzählers, seine „Sehnsucht nach dem Aufgehobensein in sinnhaften Momenten“ (FranK 2003: 224). Im Verlauf des Textes nimmt sie dann zu, die Angst, eine „halbwache Vorahnung“ von „etwas Kommendem, etwas Dunklem“ (FL: 123). Noch ist es gut versteckt, niemandem hat er davon erzählt, denn: „Es liegt hinter den Dingen, hinter den Schatten, [...] und es fliegt hinter den dunklen Vögeln am Himmel her" (FL: 123). Auch in der Erinnerung an den Strand der Insel Mykonos, der sich als von Homosexuellen besetzte FKK-Hochburg entpuppt, überkommen den Erzähler beim Beobachten eines vorbeifahrenden Dampfers solche Anwandlungen: „Es ist kein Sog mehr, kein Ohnmächtigwerden angesichts des Lebens, das neben einem so abläuft, sondern ein Stillsein. Ja genau das ist es: Ein Stillsein. Die Stille." (FL: 134) Ob das Todessehnsucht ist, bleibt dem Leser überlassen. Von dessen Deutung wird dann auch abhängen, ob das Ende der Geschichte auf dem Zürichsee als Selbstmord des Protagonisten empfunden wird.

\subsection{Das allgegenwärtige Ich}

Wie in einem Gedankenstrom, der gerade ins Bewusstsein gekommen ist, erzählt der Protagonist von verschiedensten, nicht selten banalen, 
Vorkommnissen. Die konsequente Figurenperspektive, die selten die Sicht eines anderen und nur gelegentlich das Wort eines anderen in seinen Wahrnehmungsraum dringen lässt, schafft eine Aura der Isolation um das Erzähler-Ich. Das Selbstbewusstsein des Helden bildet somit den eigentlichen Erzählgegenstand. Das ist wohl auch der Grund dafür, dass die Interpretationen von Faserland, die Charakterisierung des Protagonisten so unterschiedlich ausfällt. Dieser Held ist einfach nicht festzulegen. „Krachts Held kann einem wie ein Snob oder ein richtiges reaktionäres Arschloch (pardon) vorkommen - oder wie eine zumindest vorübergehend verlorene Seele, die gerettet werden muss.“ (RUTSCHKY 2003: 115)

Der Text ist an einen fiktiven Zuhörer gerichtet, der am Anfang indirekt angesprochen wird: „Das erkläre ich später, was ich damit meine“ (FL: 10). Dadurch wird der Eindruck hervorgerufen, dass der Erzähler schon Verlauf und Ende der Geschichte kennt.

Die ständigen sprachlichen Zurücknahmen und Einschränkungen, wie „Ich weiß nicht, nichts ist sicher, genau kann ich es nicht sagen, ich kann mich täuschen" machen die Unsicherheit des Protagonisten deutlich. Die Redeweise des Erzählers erinnert stark an das, wovon sie erzählt: sie ist elegant, unverbindlich, effektvoll und zugleich vorbehaltlich, wie eben PartyTalk. Jörg DörIng bezeichnet diesen Stil als eine Schreibweise, die „gruppenspezifischer Oralität" nachempfunden ist: durch die Nachahmung dieser ,generationsgebundenen Oralität“ gelingt die „,adäquate Beschreibungsleistung eines Ausschnittes von zeitgenössischer Wirklichkeit“ (DÖRING 1996: 231f.).

\section{4 Ästhetik der Oberfläche}

Die ungewöhnlich häufige Verwendung von Eigennamen in Faserland sticht sofort ins Auge: Jever - ein Pilsbier dieser Brauerei, Barbourjacke - eine teure wetterfeste Markenjacke der Firma Barbour, Chablis - eine Weißweinsorte aus Niederburgund, Fisch-Gosch - ein Fisch-Lokal, Traxx und P1 - bekannte Schicki-Micki-Diskotheken, Salem - ein Internat für Kinder aus wohlhabenden Elternhäusern, Sylt-die Insel ,der Reichen und Schönen“. Das sind die Namen allein auf der ersten Seite des Romans. Mit wenigen Worten wird so ein bestimmtes Milieu, ein bestimmter Lebensstil 
veranschaulicht, was sonst einen großen Formulierungsaufwand erfordert hätte. Es ist klar, dass dieser Ich-Erzähler eine Jugend der oberen Zehntausend und eine deutsche Clubkultur thematisiert.

Im Roman auftauchende real existierende Personen des öffentlichen Lebens, wie Diedrich Diedrichsen (Poptheoretiker), Isabella Rossellini (Model, Schauspielerin), Matthias Horx (Trend- und Zukunftsforscher) oder Wim Wenders (Filmregisseur) verstärken die besondere Gegenwartsbezogenheit. All dies kann in einem sonst eher konventionell erzählten Roman als etwas wirklich Neues in der deutschen Literatur betrachtet werden. In Faserland sind an die 70 Marken- und Produktbezeichnungen zu zählen. Besonders häufig tauchen auf: Namen von Autotypen (Ferrari, Golf, Mercedes, Porsche), ohne dass der Held ein Autonarr ist, von Getränken (Jack Daniels, Jever, Pernod) und Genussmitteln (Hanuta, Lindt, Marlboro), denen der Held schon eher zuspricht. Typisch sind Markennamen aus dem Medienbereich (z.B. Bravo, Stern, Süddeutsche, Tempo) und der Modebranche (Armani, Jil Sander, Rolex, Swatch, Tiffany). Alle diese Warenund Produktbezeichnungen sind zum einen authentische, dokumentarische Elemente und haben zum anderen eine symbolische Bedeutung als Repräsentationen des Zeitgeistes, der Schicki-Micki-Szene, des Schnösel- und des Reichtums (vgl. GraBIENSKI 2001). So ist die Barbourjacke zum Kultobjekt (nicht nur) des Erzählers geworden, eben weil sie mehr ist als eine edle wetterfeste Wachslederjacke mit per Reißverschluss abtrennbarem Innenfutter und anderen diversen Bequemlichkeiten.

Viele Beobachtungen werden von Erzähler einer ästhetischen Bewertung unterzogen. Im Text stehen nebeneinander banale, alltägliche Marken wie etwa Ehrmann-Joghurt oder Ballisto-Riegel, mit denen sich der Protagonist am Flughafenbüfett die Taschen voll stopft, neben Luxusgütern wie der Cartier-Uhr und dem Tiffany-Notizblock, die er bei der alten Sitznachbarin im Flugzeug sieht. Der Erzähler wird nicht müde, immer wieder auf den einzig wichtigen Unterschied zwischen dem Schönen (oder wie er es formuliert: dem „Feinen“) und dem Hässlichen zu verweisen. Tee mit Milch hat z.B. einen Geruch, der ,so fein nach Zuhause riecht und nach Sicherheit" (FL: 117) und Hannah, eine Bekannte, die er in einem Münchner Lokal bei der Arbeit beobachtet, lächelt jeden an, ,auch die Blöden und die Arschlöcher und die Aufdringlichen. [...] Hannah ist so gut und so fein zu denen, dass es mir fast weh tut.“ (FL: 111) „Weiße Zähne“" 
sind es auch, die ihn anziehen, nicht nur bei den Alitalia-Piloten in der Kindheitserinnerung (FL: 47), sondern sogar, wenn jemand ihm nicht auf Anhieb sympathisch ist: Eugen z.B. ,[...] hat ja ein gutes Jackett an, [...] und er hat weiße Zähne“. (FL: 92) So detailversessen der Erzähler das Schöne lobt, so heftig kann er auch das Hässliche verdammen. Frankfurt ist z.B. „extrem abstoßend“ und auch der „Bord-Treff“ im Zug ist „unfassbar hässlich“ und ,grauenvoll [...] gestaltet“ (FL: 78). Hässlich sind im Grunde auch alle alten Leute: ,sie haben alle überdimensionale Nasen und Ohren, weil ja Nasen und Ohren im Alter immer weiter wachsen“" (FL: 89). Auch den Freund Nigel trifft diese Bewertung, als der Protagonist feststellt, dass er „immer ein bisschen schäbig angezogen ist, nicht so direkt schäbig“, aber ,irgendwie schlampig“ (FL: 27). Hässlich sind Künstler, zumindest die die sich absichtlich schlecht anziehen: „So mit Cordoverall, [...] dicken Turnschuhen, fettigen Haaren und Arbeiterkappe.“ (FL: 68)

\section{Zwischen Pop und Nicht-Pop}

Doch was ist eigentlich Pop an Krachts Roman Faserland, der als Initiationsroman der neuen Popliteratur gehandelt wird? Thomas Jung (2002: 51) nennt für dieses Literaturgenre folgende Kriterien:

(a) tradierte allgemein gültige Wahrheits- und Legitimierungsdiskurse (Klasse, Rasse, Geschlecht, Geschichte etc.) werden in Frage gestellt;

(b) der Adressat ist ein breites, soziologisch heterogenes Lesepublikum, nicht der bildungsbürgerliche Rezipient;

(c) Thematik wie Darstellung zielen auf die Darstellung von Jugendlichkeit bei Produzent wie Rezipient;

(d) Thematik wie Darstellung zielen auf Lebensintensität bzw. Hedonismus in der Haltung der Figuren;

(e) Thematik wie Darstellung zielen auf das „Peinliche“ bzw. auf die Amoralität;

(f) die Erzählhaltung der Figuren ist oftmals eine apolitische (im Sinne traditioneller Diskurse von Parteipolitik);

(g) die Darstellungsformen basieren auf der Wiederverwertung- und Vermarktung - sowie auf der sukzessiven Nivellierung von spezifischen Kulturtraditionen, Motiven und Strukturen. 
Sucht man nach solchen Merkmalen im Roman Faserland, wird man schnell fündig. Die Jugendlichkeit des Autors (Kracht ist Ende Zwanzig, als der Roman erscheint) und des Protagonisten (etwa Anfang bis Mitte Zwanzig) sowie die (zumindest auf der Oberfläche) jugendgemäße Thematisierung von Partys, Musik und Drogen treffen auf diesen Roman zu. Der Roman erreicht durch die erwähnten Markennamen und durch die Nennung von Personen des öffentlichen Lebens eine Gegenwartsbezogenheit, wie sie für Poptexte typisch ist. Es kann davon ausgegangen werden, dass der Autor beim Schreiben nicht an einen „bildungsbürgerlichen“ Leser gedacht hat, sondern an eine „breite Masse“ junger Leute zwischen Mitte Zwanzig und Mitte Dreißig. Dabei offenbart der Protagonist hin und wieder sein Halbwissen. Er erzählt, sein Freund Alexander sähe ,irgendwie mittelalterlich aus, wie auf einem Bild von Walter von der Vogelweide oder Bernhard von Clairvaux. Das sind beides mittelalterliche Maler, das weiß ich“. (FL: 63)

Politisch interessiert wirkt der Held dieses Romans nicht gerade (zumindest nicht im Sinne von Parteienpolitik), dennoch scheint ihn die deutsche Vergangenheit sehr zu beschäftigen. Neu ist dabei die Art der Einbindung historischer Motive in den Text. Während einer frühmorgendlichen Taxifahrt denkt der Erzähler: „Hamburg wacht auf, [...] und dann muss ich plötzlich an die Bombennächte im Zweiten Weltkrieg denken und an den Hamburger Feuersturm [...] und ich würde gerne mit dem Taxifahrer darüber reden, aber er hat Mundgeruch" (FL: 43). Das sind keine Provokationen im großen Stil, nur kleine spitze Bemerkungen und unvermittelte Ausbrüche eines gelangweilten Dandys. Degoutant ist aber die Haltung des Protagonisten, als er in Kilchberg bei Zürich das Grab Thomas Manns sucht und dabei einen Hund beobachtet, der über den nächtlichen Friedhof streunt: „Ich finde das blöde Grab von Thomas Mann nicht. [...] Der Hund kackt tatsächlich auf eines von den Gräbern.“ Ihm kommt der Gedanke, „dass der Hund vielleicht auf Thomas Manns Grab gekackt haben könnte“ (FL: 152 f.).

Wirklich „popliterarisch“ wiederum ist die häufige Darstellung des „Peinlichen“ in Faserland, wie in der Episode mit dem furzenden Taxifahrer, der während der Fahrt Zigaretten anbietet und bloß redet, „damit wir nicht mehr an sein Gefurze denken“ (FL: 33).

Von „Lebensintensität und Hedonismus“ kann dagegen bei dem Protagonisten dieses Romans nicht die Rede sein. Die besuchten Partys 
und die Situation des Reisens scheinen von Lebensintensität zu zeugen, doch kann der Protagonist weder etwas genießen noch sein Glück finden. Auf der Hamburger Party, als Nigel aus seinem Tütchen eine Pille nimmt und sie mir in die Hand drückt, denke ich: $\mathrm{Na}$ ja, ich kann das ja mal versuchen. [...] im Grunde finde ich Drogen absolut widerlich, aber ich stecke mir das Ding in den Mund“ (FL: 37). Später fühlt er sich „,so richtig körperlich ausgelaugt" (FL: 42). Als er seinen Freund Nigel mit zwei anderen Partygästen im Bett überrascht, verlässt er fluchtartig die Wohnung: „Auf dem Weg aus der Stadt [...] fange ich an zu heulen.“ (FL: 46) Auch die nicht nur durch Unmengen Alkohol verursachte wiederkehrende Übelkeit bis zum Erbrechen, die den Protagonisten plagt, ist Indiz dafür, dass er eigentlich verzweifelt ist.

Von literaturwissenschaftlicher Seite gibt es die unterschiedlichsten Ansätze die neue Popliteratur und ihre Bedeutung zu untersuchen. So versteht der Pop-Theoretiker Moritz BASSLER diese neue literarische Strömung als „Phänomen eigenen Rechts“. Seiner Auffassung nach speichern die neuen Pop-Autoren gewitzt-provozierend Banalitäten in einer Art kulturellem Archiv. Der „,bisherigen Erfolgsliteratur stehen vielfältige Verfahren der Katalogisierung und Listenbildung gegenüber, die sich ideal als Werkzeuge zur ersten Archivierung eignen“ (BASSLER 2002: 186). Bei Kracht wird man dagegen vergeblich nach Listen oder Katalogen von „profanen Dingen“ suchen, die man in den Romanen Benjamin von Stuckrad-Barres oder bei Florian Illies finden kann. In Faserland werden Markenartikel nicht listenweise genannt, von „wildwucherndem Assoziieren" kann nicht die Rede sein.

Für Katharina RutschKy ist Goethes Jugenderfolg Die Leiden des jungen Werther das „Urbild des Popromans“, wo Goethe „für das Recht des jugendlichen Einzelnen auf eine eigene Sicht der Welt warb“. Goethe war nie wieder so ein Erfolg beschieden, denn der Poproman ist „ein Solitär“ (Rutschky 2003: 108). Auch der Protagonist aus Faserland berichte „aus jenem Zwischenreich von Jugendalter, Versorgung und Ausbildung, in dem der Poproman immer angesiedelt ist". (Rutschky 2003: 113) Freilich sei die Liebe als zentrales Motiv in Faserland nicht so präsent, wie in Goethes Jugendroman. Vielmehr zeige sie sich in der ,negativen Fassung, also der vollständigen Lieblosigkeit und Leere, die Krachts Alter ego in die Welt 
projiziert“ (RutschKy 2003: 116). Carsten Gansel fasst es gleich so zusammen: „Die neue deutsche Pop-Literatur ist in ihrem Kern Adoleszenzliteratur.“ (GANSEL 2003: 236)

Für eine „Coming-Out-Geschichte“ hält Mathias Mertens den Roman: „Der 11-jährige Knabe, der mit blutigem Bein an einen Mann gefesselt ist, der später im Bett bei einem Mädchen nur exkremieren kann, der heute genauso aussieht wie sein Freund Nigel und diesem auch auf seiner Reise ständig begegnet, der die Jacke mit einem anderen Freund tauscht, der mit einem dritten Freund zu dessen Selbstmord fährt, der in Zürich das Grab Thomas Manns sucht und der schließlich in goethescher Symbolik zum „anderen Ufer“" des Sees aufbricht.“ (Mertens 2003: 208) Auch wenn eine solche Hypothese nicht ganz abwegig ist, ist die Argumentation oberflächlich und durch „ungenaues“ Lesen zurechtgebogen: Gefesselt ist er bei einem Hüpfspiel, wo er sich durch Übereifer das Bein blutig wetzt; im Gästezimmer des Elternhauses der Freundin übernachtet der betrunkene Erzähler ganz allein; Nigel begegnet er höchstens zwei Mal; „schlampig“ wie Nigel sieht er nie aus; Alexanders Barbourjacke stiehlt er; die Nachricht von Rollos Selbstmord erfährt er erst später. Die in zahlreichen Situationen deutlich werdende Homophobie des Protagonisten könnte (als Phase eines Coming-Outs) die obige Hypothese dagegen stützen. Szenen, in denen er sich zu Mädchen hingezogen fühlt oder mit ihnen flirtet (z. B. FL: 40 f.), scheinen gebrochen: Als ein Mädchen auf sein „charmanteste[s]“ Lächeln reagiert, läuft ihm "ein kleiner angenehmer Schauer den Rücken herunter, der gleiche Schauer übrigens, den ich auf öffentlichen Pissoirs bekomme, wenn ich auf die Duftwürfel pisse.“ (FL: 76) Merkwürdig ist auch seine Reaktion auf eine fast zufällige Annäherung: „Unterwegs streift Karins Hand ganz kurz meine Hand, und ich bekomme einen Hustenanfall.“ (FL: 16)

Gerade die Vieldeutigkeit oder Offenheit vieler Szenen und Passagen machen wohl den Reiz des Buches aus. Dirk Frank hält dieses „Mehrdeutigkeitskalkül“ gar für eines der auffälligsten Merkmale des Romans und meint, eine prinzipielle vom Protagonisten ausgehende „Verweigerung sei das Bewegungsgesetz der Geschichte“. In diesem Sinne zieht der Held am Ende des Romans auch ein Verschwinden „einem wie auch immer gearteten Dagegen- oder Dafürsein vor“" (Frank 2003: 226). 


\section{Der Roman 1979}

\subsection{Eine Reise in die Dunkelheit}

Krachts zweiter Roman 1979 besteht aus zwei Teilen. Die ersten sieben Kapitel sind unter der Überschrift „Iran, Anfang 1979“ zusammengefasst. Der Schauplatz ist Teheran; den Hintergrund bilden die realen Geschehnisse dieser Zeit wie der Sturz des prowestlichen Regimes des Schahs von Persien und die Gründung eines fundamentalistischen Religionsstaates durch Ayatollah Khomeini. Der zweite Teil des Romans umfasst die Kapitel acht bis zwölf und ist mit „China, Ende 1979“ überschrieben. Die erzählte Zeit beträgt also etwa ein Jahr. Schauplätze im zweiten Teil sind das von China besetzte Tibet mit dem heiligen Berg Kailasch und ab Kapitel zehn chinesische Arbeitslager

Der Erzähler, ein Innenarchitekt und sein hoch gebildeter aber gesundheitlich angeschlagener Freund Christopher, reisen durch den Iran nach Teheran, wo schon die Panzer an den Straßenkreuzungen stehen. Die zwei jungen Deutschen, ein homosexuelles Pärchen, gehören der internationalen Partyszene an, die sich zur Kreuzfahrt in der Ägäis, an der französischen Mittelmeerküste oder eben in den Villen im reichen Norden der persischen Hauptstadt trifft. Sie sind eingeladen zu einer Party. Im Garten der Villa amüsieren sich die Gäste bei Alkohol und Drogen. Der Erzähler trifft dort Mavrocordato, einen mysteriösen rumänischen Intellektuellen, der ihm prophezeit, dass er sich in wenigen Tagen halbieren werde. Kurze Zeit danach fällt Christopher in eine Glastür der Villa und stirbt Stunden später in einem schäbigen Krankenhaus. Die Revolution bricht aus. Der Protagonist irrt durch Teheran und trifft wieder auf Mavrocordato, der ihm zu einem seelenreinigenden Gang um den heiligen Berg Kailasch im Tibet rät. Tatsächlich macht sich der Erzähler auf den Weg dorthin, um dort zu erkennen: „Die Reinwaschung [...] war einfach nicht passiert.“ (1979: 141) Der Pilger wird kurz darauf von chinesischen Soldaten aufgegriffen, die ihn zu einem russischen Spion erklären und in Arbeits- und Umerziehungslagern internieren. In der Wüste Lop Nor, wo Protagonist - das westliche Individuum - gemeinsam mit anderen Häftlingen unter unmenschlichen Bedingungen die Wüste urbar machen. 


\subsection{Der Protagonist - ein Medium "wide open"}

Im Unterschied zu dem Protagonisten in Faserland ist dieser Erzähler nur noch „leer und ganz ohne Vergangenheit [...], als ob alles, was vorher war, ausgelöscht worden wäre, jeder Geruch oder jede Farbe“ (1979: 34). Mavrocordato bezeichnet ihn als „wide open“ und sagt: „Sie haben sich [...] Ihre Unschuld bewahren können, Ihre Naivität“ (1979: 59 f.); Christopher hingegen meint, ,ich sei etwas dämlich, womit er ja auch vielleicht recht hatte" (1979: 19). Er registriert, aber er versteht nicht und hinterfragt nicht. Die unpräzise Sprache in Faserland, die an mündliche Rede erinnert, ist hier einem schlichten und klaren Erzählstil gewichen. In einem unbeteiligt wirkenden Tonfall berichtet er dem Leser genauso präzise von der ästhetisch ansprechenden Inneneinrichtung der Teheraner Villa wie vom Tod seines Freundes oder dem Leben in einem chinesischen Straflager. Nur kurz bewegt ihn das Ende seines Freundes: „es war so wenig schick“ (1979: 78). Immerhin kommt er zu dem Schluss: „Irgend etwas muss sich ändern“ (1979: 79). Dies ist die einzige Stelle im Text, an der eine Entwicklung des seelisch-moralisch verkümmerten Protagonisten sichtbar wird. Innerhalb seiner Beziehung zu Christopher, die schon lange zerrüttet ist, hat der Erzähler eine passive, unterwürfige Rolle: „Ich drehte mich etwas zur Seite, so dass ich ihm meinen Hals als Angriffsfläche hingab. Dies war die Geste, die ich oft benutzte, um ihn zu besänftigen, ohne dass er die Geste als solche erkannte.“ (1979: 30)

Die aus Faserland bekannte Übelkeit ereilt den Protagonisten in 1979 schon im ersten Satz: ,Auf dem Weg nach Teheran sah ich aus dem Autofenster, mir wurde etwas übel“" (1979: 17). Sonst eher unpolitisch und nahezu blind gegenüber der politischen Umbruchsituation in Teheran, wird ihm beim Anblick eines Polizisten, der „hinkniete und die Füße eines Geistlichen küsste“, so übel, dass er sich „dabei fast übergeben musste“ (1979: 94).

Das Grauen in den chinesischen Arbeitslagern wird in einer regungslosen und demütigen Sprache geschildert: „Ein paar Häftlinge waren mongoloid; [...] irgendwann verschwanden sie alle, [...]. Ein Gefangener flüsterte mir zu, man habe ihre Organe gebraucht, was ich nicht verstand. Ich fragte auch nicht nach.“ (1979: 176) Der Protagonist ist nur noch ein Medium, durch das Andere sprechen, das Andere beliebig mit Sinn füllen. Er, von dem Mavrocordato gesagt hatte, er sei „ein offenes Gefäß, wie der 
Kelch Christi““ (1979: 60), wird so zu einer Art Märtyrerfigur, die stellvertretend für die Sünden des westlichen Menschen Buße tut. Gefordert wurde es vorher an mehreren Stellen, so z.B. von Massoud, dem Besitzer eines Cafés, der sich auf den Koran beruft: „,Wir haben uns alle verschuldet, weil wir Amerika zugelassen haben. Wir müssen alle Buße tun. Wir werden Opfer bringen müssen, jeder von uns.“ (1979: 98) Der Protagonist ist dennoch kein Märtyrer im eigentlichen Sinne, denn es fehlt ihm die zentrale Kraft des Märtyrers - der Glaube.

\subsection{Ende der Pop-Ästhetik}

Das Verhältnis des Erzählers zu Äußerlichkeiten, zu den schönen Dingen, lässt sich mit dem des Protagonisten in Faserland vergleichen. Doch erscheinen popkulturelle Elemente wie Markenartikel und Musik in dieser Umgebung eher bloßgestellt. „Hermès, Paris“, sagt Massoud zu einer kleinen schwarzen Tasche, die aussieht wie ein Arztkoffer. Doch es ist nur „ein kleiner halbintellektueller, persischer Scherz“ (1979: 100). Im islamistischen Iran und im kommunistischen China hört die Popkultur auf. Unter den Band-Namen (vgl. Blondie, Devo, nehmen die schon in Faserland erwähnten Ink Spots (eine schwarze Band aus den vierziger Jahren; FL: 135) einen besonderen Platz ein. Auf der Ink-Spot-Kassette, die der Erzähler von Hasan (dem Fahrer) geschenkt bekommt, sind, wie sich später herausstellt, die nach Persien geschmuggelten Reden Khomeinis.

Markenprodukte werden nur noch sporadisch genannt, wie u.a. eine Lalique-Schale, Unterhosen von Brooks-Brothers, Christophers PaisleyEinstecktuch und die Berluti-Schuhe. Gerade die Berluti-Schuhe zeigen, wie der Faserland-Luxus in 1979 abzubröckeln beginnt. „Die besten Schuhe der Welt“ (1979: 127) zerfallen nach und nach, der Held tauscht sie gegen die von seinem Reiseführer selbstgebastelten Filzschuhe ein.

Peinliche oder kuriose Situationen, wie in Faserland, gibt es in 1979 nicht mehr. Exkremente kommen auch in diesem Roman vor, doch beschreibt der Erzähler nur noch die erschütternde Realität. In Faserland amüsiert er sich auf der Zugtoilette noch bei dem Gedanken, wie früher „die Scheiße“ aus den Toiletten „direkt auf den Kopf oder auf die Plastikmöbel im Garten“ der Menschen fiel (FL: 23 f.). In 1979 sind es die 
Wände des schäbigen Krankenzimmers, die „mit Kot und Blut beschmiert“ (1979: 73) sind. Und im chinesischen Lager schließlich läuft, ,wegen der Ernährung [...] der Durchfall [...] den Gefangenen dann die Beine herunter [...] auf die Holzpantinen“ (1979: 172), so dass diese auf die Idee kommen, wegen des Proteingehalts „vom Müllhaufen des Lagers Maden zu stehlen, um diese dann heimlich [...] mittags in die dünne Suppe zu legen. Auf der Müllkippe [...] lag ohnehin nicht viel. Das meiste war menschlicher Kot, zerfetzte Kleidung, ausgekochte Kohlstrünke [...]“ (1979: 180).

\subsection{Spurensuche im Orient}

Ohne sich überdeutlich aufzudrängen, machen unzählige Andeutungen, Signale und Hinweise das Buch zu einer Fundgrube für den gründlichen Leser. So liegt das Bündel Geld, das der Erzähler für seine Reise erhält, in einem Buch des Soziologen Karl Mannheim, der den Typus eines über jegliche Ideologien erhabenen und einer höheren Moral verpflichteten Intellektuellen entwarf. Mavrocordato mit seinen nackten, stark behaarten Füßen, seinem Haarschopf, der senkrecht in die Luft hochragt, und seinen seherischen Fähigkeiten (1979: 50) erscheint als wahrhaft fantastische Figur. Dunkle Speisen, wie er sie dem Protagonisten vorsetzt, „Schwarzhirsch mit Pflaumensauce“ und „Blutpudding mit Brombeeren“ (1979: 104), werden schon in Joris-Karl Huysmans Roman Gegen den Strich gegessen. Der Rumäne Mavrocordato erzählt von seinem Großvater, der an der Schwarzmeerküste, zeitgleich wie der Dichter d'Annunzio mit „Fiume“, einen utopischen Kleinstaat gründete. „Mavrocordato“ ist übrigens auch der Name eines griechischen Freiheitshelden, eines späteren Begleiters Lord Byrons und Mary Shelleys.

Der weit gereiste Kracht lässt sich in eine Traditionslinie einordnen, die bei Hugo von Hofmannsthal im Jahre 1906 mit dem Essay „Tausendundeine Nacht“ beginnt, sich fortsetzt in der Reiseliteratur der 30er-Jahre einer Annemarie Schwarzenbach, Ella Maillart und Robert Byron, der ein autobiografisches Reisetagebuch, Road to Oxania schrieb (von dem Kracht einiges in den Roman übernommen hat), und schließlich über Paul Bowles, den Kracht als Vorbild nennt, bis hin zu Bruce Chatwin führt, der auf den Spuren des von ihm verehrten Robert Byron Persien durchquert. 


\section{5 Überleben in Finsternis}

Während den Ich-Erzähler in Faserland ein diffuses Gefühl der Resignation bestimmt und ihn ,dunkle Ahnungen“ bedrängen (FL: 123), trifft er doch immerhin am Ende die Entscheidung, „die große Maschine“ Deutschland (FL: 149) zu verlassen. Der Erzähler von 1979 nimmt dagegen alles nur noch hin und ist am Ende zu keiner wirklichen Entscheidung mehr fähig. Hier wie dort verweigert Kracht seinen Figuren eine Perspektive. Im Gegenteil, die physischen wie psychischen Mechanismen der Zersetzung des Individuums durch ein totalitäres Regime wie das hier vorgeführte chinesische müssen früher oder später zur Auslöschung des Menschen führen: „Ich wog nur noch halb so viel wie früher, [...] 38 Kilo stand auf der weißen Keramikwaage. Ich müsse nun kein Blut mehr geben, ich sei viel zu dünn und schwach, sagte der Arzt, aber ich tat es trotzdem, freiwillig." (1979: 182 f.) Der psychische Verfall zeigt sich auch in der befremdlichen Freude über den starken Gewichtsverlust: „Ich dachte an Christopher, daran, dass ich mich immer zu dick gefühlt hatte, und ich war glücklich darüber, endlich seriously abzunehmen.“ (1979: 166) Als der Protagonist, um nicht zu erfrieren, in seiner Zelle ein Feuer anzündet, ärgert er sich über seine Aufmüpfigkeit: „Schließlich hatten sie nur ihre Pflicht getan.“ (1979: 153)

Die Arglosigkeit, mit der er seine Situation hinnimmt, erschüttert. Nachdem der Erzähler fünf Nächte an einen nicht funktionierenden Heizkörper gekettet war, hat er nichts weiter zu beanstanden als: „Ich hatte eine schlechte Gesichtshaut bekommen. Nach der Rasur hatte man mir kein Kölnisch Wasser gegeben, so dass jetzt meine gesamte untere Gesichtshälfte mit kleinen roten Pusteln übersät war.“ (1979: 153 f.)

Am Ende der Geschichte führt Kracht, untermalt durch die Charakteristik der Landschaft, eine apokalyptische Untergangs- und Auslöschungsvision vor: „Nicht einmal Vögel waren am Himmel zu sehen, der Ort, an dem wir und Tausende anderer Menschen lebten, war ausgestorben, so leblos wie die Oberfläche des Mars. Wir waren verschwunden, es gab uns nicht mehr, wir hatten uns aufgelöst.“ (1979: 181) Das Individuum mit seinen speziellen Interessen und stilisierten Lebensformen zählt hier nichts mehr. Was bleibt, ist die unterwürfige Anpassung an die zwangskollektivierte Lagerhaltung. Die letzten Sätze des Protagonisten sind: „Ich war ein guter Gefangener. Ich habe immer versucht, 
mich an die Regeln zu halten. Ich habe mich gebessert. Ich habe nie Menschenfleisch gegessen.“ (1979: 183).

\section{Literaturverzeichnis}

\section{Primärliteratur}

KRACHT, Christian. Faserland. Köln, Goldmann-Taschenbuch 1997. [im dtvTaschenbuch von 2002 finden sich die Zitate jeweils 4 Seiten weiter; zitiert: FL]

KRACHT, Christian. 1979. Köln, Kiepenheuer \& Witsch 2001. [seitenidentisch mit dem dtv-Taschenbuch von 2003; zitiert: 1979]

\section{Sekundärliteratur}

Bassler, Moritz. Der Deutsche Pop-Roman. München, C. H. Beck Verlag 2002.

BLANKE, Ludger „Herzlose Finsternis“. Rezension in: Jungle World 24. 10. 2001.

DöRING, Jörg. „Redesprache, trotzdem Schrift - Sekundäre Oralität bei Peter Kurzeck und Christian Kracht“. In: Jörg DÖRING / Christian JäGER / Thomas Wegmann (Hg.). Verkehrsformen und Schreibverbältnisse. Medialer Wandel als Gegenstand und Bedingung von Literatur im 20. Jahrbundert. Opladen, Westdeutscher Verlag 1996.

Frank, Dirk. „Die Geburt des „Tristesse Royale“ aus dem Geiste der achtziger Jahre. In: Text + Kritik X / 03, Sonderband Pop-Literatur. München, Verlag Text + Kritik 2003. 218-233.

Freund, Wieland. „Aufpoliert“. In: Berliner Morgenpost 05. 10. 2003.

Gansel, Carsten. „Adoleszenz, Ritual und Inszenierung in der Popliteratur“. In: Text + Kritik X / 03, Sonderband Popliteratur. München, Verlag Text + Kritik 2003. 234-257.

GrabIENSKI, Olaf. „Christian Krachts Faserland - Eine Besichtigung des Romans und seiner Rezeption“. 2001. www.olafski.de/arbeiten/kracht.pdf 
Heilbrunn, Jacob. „Der Ernst des Lebens - Ein Abgesang auf die deutsche Popliteratur“. In: Süddeutsche Zeitung - Magazin. 05. 10. 2001.

Henning, Peter. „Kippfiguren“. Rezension in: Die Weltwoche 18. 10. 2001.

ILLIES, Florian. Generation Golf. Frankfurt am Main, Fischer-Verlag 2000.

JACOB, Günther. „Gelingen der Nation“. In: konkret Nr. 26, 2001/2002, 10 -13 .

JäHNER, Harald. „Dandys Straflager“. Rezension in: Berliner Zeitung 09. 10. 2001.

Krumbholz, Martin. „Polierte Oberfläche“. Rezension in: Freitag 24. 03. 1995.

Mertens, Mathias. „Christian Kracht, Benjamin v. Stuckrad-Barre und ihre mutmaßlichen Vorbilder“. In: Text + Kritik X / 03, Sonderband PopLiteratur. München, Verlag Text + Kritik 2003, 201-217.

Rutschky, Katharina. „Wertherzeit“. Der Poproman - Merkmale eines unerkannten Genres. In: Merkur, Stuttgart 02 / 2003, 106-117.

Schмттт, Michael. „Produkt-Realismus“ - Christian Krachts Début Faserland. In: Neue Züricher Zeitung 04. / 05. 03. 1995.

SIEBER, Peter. Parlando in Texten. Zur Veränderung kommunikativer Grundmuster in der Schriftlichkeit. Tübingen, Niemeyer 1998.

Vormweg, Christoph (1995): „Trübe Erben“. Rezension in: Süddeutsche Zeitung, Literaturbeilage 06. 04. 1995.

Ziegler, Helmut (1995): „Christian Kracht. Faserland“. Rezension in: Die Woche. 13 / 1995. 